\title{
Impact of the systemic immune-inflammation index for the prediction of prognosis and modification of the risk model in patients with metastatic renal cell carcinoma treated with first-line tyrosine kinase inhibitors
}

Jun Teishima ${ }^{1}$; Shogo Inoue ${ }^{1}$; Tetsutaro Hayashi ${ }^{1}$; Akio Matsubara ${ }^{1}$; Koji Mita ${ }^{2}$; Yasuhisa Hasegawa ${ }^{3}$; Masao Kato ${ }^{4}$; Mitsuru Kajiwara ${ }^{5}$; Masanobu Shigeta ${ }^{6}$; Satoshi Maruyama $^{7}$; Hiroyuki Moriyama ${ }^{8}$; Seiji Fujiwara ${ }^{9}$

${ }^{1}$ Department of Urology, Graduate School of Biomedical and Health Sciences, Hiroshima University, Hiroshima, Japan; ${ }^{2}$ Department of Urology, Hiroshima-City Asa Citizens Hospital, Hiroshima, Japan; ${ }^{3}$ Department of Urology, Fukuyama Medical Center, Fukuyama, Japan; ${ }^{4}$ Department of Urology, Hiroshima General Hospital, Hatsukaichi, Japan; ${ }^{5}$ Department of Urology, Hiroshima Prefectural Hospital, Hiroshima, Japan; ${ }^{6}$ Department of Urology, Kure Medical Center and Chugoku Cancer Center, Kure, Japan; ${ }^{7}$ Department of Urology, Miyoshi Central Hospital, Miyoshi, Japan; ${ }^{8}$ Department of Urology, Onomichi General Hospital, Onomichi, Japan; ${ }^{9}$ Department of Urology, Higashi-Hiroshima Medical Center, Higashi-Hiroshima, Japan

Funding: This study was supported by Pfizer Inc. and Novartis Pharma Inc.

Acknowledgement: In addition to the authors, the following investigators participated in the study: Shuntaro Koda, Kure Medical Center; Hiroshi Masumoto, Fukuyama Medical Center; Shinya Ohara and Kosuke Sadahide, Hiroshima Prefectural Hospital; Koichi Shoji, Mazda Hospital; Hideo Iwamoto, Higashi-Hiroshima Medical Center; Hideki Mochizuki and Daiki Murata, Hiroshima City Asa Citizens Hospital; and Yuichi Kadonishi, Onomichi General Hospital.

Cite as: Can Urol Assoc J 2020 June 5; Epub ahead of print. http://dx.doi.org/10.5489/cuaj.6413

Published online June 5, 2020

$* * *$ 


\begin{abstract}
Introduction: International Metastatic Renal Cell Carcinoma Database Consortium (IMDC) criteria are the most representative risk model for patients with metastatic renal cell carcinoma (mRCC). However, the intermediate-risk group of IMDC criteria is thought to include patients with different prognoses because the majority of the patients are classified into the intermediate-risk group. In this study, we investigated the impact of systemic immune-inflammation index (SII), which is calculated based on neutrophil count, platelet count, and lymphocyte count, on predicting the prognosis in patients with $\mathrm{mRCC}$ and its usefulness for re-classification of patients with a more sophisticated risk model.
\end{abstract}

Methods: From January 2008 to January 2018, 179 mRCC patients with a pretreatment and SII were retrospectively investigated. All patients were classified into either a highSII group or a low-SII group based on the cutoff value of a SII at 730, as reported in previous studies; the overall survival (OS) rates in each group were compared.

Results: The median age was 65 years old. Males and females comprised 145 and 34 cases, respectively. The categories of favorable-, intermediate-, and poor-risk groups in the IMDC model were assessed in 39, 102, and 38 cases, respectively. The median of the observation period was 24 months. The low-SII and high-SII groups consisted of 73 and 106 cases, respectively. The 50\% OS in the high-SII group was 21.4 months, which was significantly worse than that in the low-SII group (49.7 months; $\mathrm{p}<0.0001$ ).

Multivariate analysis showed that a high SII was an independent predictive factor for a worse OS. Next, we constructed a modified IMDC risk model that included the SII instead of a neutrophil count and a platelet count. By using this modified IMDC model, all cases were re-classified into four groups of 33, 52, 81, and 13 cases with $50 \%$ OS of $88.8,45.9,29.4$, and 4.8 months, respectively.

Conclusions: The systemic immune-inflammation index is useful for establishing a more sophisticated prognostic model that can stratify mRCC patients into four groups with different prognoses. 


\section{Introduction}

While the spread of screening has led to an increase in the detection of clinically localized renal cell carcinoma (RCC), more than $30 \%$ of patients with RCC have metastases at initial presentation [1]. Introducing targeted agents, especially tyrosine kinase inhibitors (TKIs), has led to improved prognosis of metastatic RCC (mRCC) in the past decade [2]. In addition, some randomized control trials showed the effectiveness of immune checkpoint inhibitors to mRCC patients [3, 4]. Several guidelines recommend the choice of a first-line agent based on the risk model $[5,6]$.

The International Metastatic Renal Cell Carcinoma Database Consortium (IMDC) risk model [7] was one of the most widely used for mRCC. This model is useful in the real world of clinical practice, and its use has spread all over the world. However, patients with relatively different prognoses are thought to be classified into an intermediate risk group because it consists of more than half of patients. Establishing a more sophisticated prognostic model is a very important objective.

Several molecules or indexes related to the inflammatory response derived from blood samples have been demonstrated as candidates of biomarkers predicting the effect of the treatment or prognosis of cases with $\mathrm{mRCC}$ regardless of therapeutic options [8]. Two of six parameters of the IMDC prognostic model, neutrophil count and platelet count, are also involved in the inflammatory response. The systemic immune inflammatory index (SII) was defined as follows: platelet count $\times$ neutrophil count / lymphocyte count [9]. SII has been reported as a prognostic marker for several malignant diseases, and it can represent the balance of inflammation and immune response of hosts [10]. We investigated the prognostic impact of SII in patients with mRCC treated with $1^{\text {st }}$-line TKI and assessed the modified IMDC risk model using SII.

\section{Methods}

\section{Patients}

From January 2008 to January 2018, 179 patients with pathologically diagnosed mRCC treated with TKI as $1^{\text {st }}$-line agents at our institute and affiliated hospitals in Hiroshima Prefecture in Japan were retrospectively investigated after approval by the Ethical Committee of Hiroshima University (Allowance notification number; E-45). The SII 
was calculated based on the data upon starting the prescription of $1^{\text {st }}$-line targeted agents. The cut-off value of the SII was determined to be 730, as reported in previous studies [10]. Cases with pretreatment SII were 730 or higher, and the others were classified into a "high SII group" or a "low SII group," respectively.

We compared the clinical and pathological data including age, sex, histological finding, metastasis status, choice of drug, prior nephrectomy, Karnofsky performance status (KPS), anemia, serum calcium, neutrophil count, platelet count collected for all patients, and the distribution of these parameters in each group. The overall survival (OS) of each group classified according to these parameters was analyzed.

The modified IMDC model was determined using five poor prognostic factors including KPS $<80 \%$, time from diagnosis to treatment $<1$ year, anemia, hypercalcemia, and SII $>730$. Cases were classified into 4 groups based on the presence of the number of these factors, $0,1,2$ to 3 , or 4 to 5 , respectively.

\section{Statistical analysis}

The differences in the distribution of variables among groups were evaluated using a chi-square test for categorical variables and a Mann-Whitney test for continuous variables, respectively. Pearson correlation coefficients were assessed between SII and other inflammatory parameters. Tumor responses were determined using an investigator assessment according to the response evaluation criteria in solid tumor (RECIST) version 1.1. The OS was determined using the Kaplan-Meier method, and the differences between groups were analyzed using log-rank testing. Multivariate analyses of parameters associated with OS were evaluated using the Cox proportional hazards regression model. All statistical analyses were conducted using the JMP 10.0.0 (SAS Institute Inc., $\mathrm{NC}$ ), and $\mathrm{p}$ values less than 0.05 were considered to be statistically significant.

\section{Results}

One hundred seventy-nine patients with $\mathrm{mRCC}$ treated with $1^{\text {st }}$-line TKI were investigated in this study. The SII was 94.6 to 4603.1 (median 609.7), and positive correlations were found between the SII and other inflammatory parameters including C-reactive protein (CRP), neutrophil count, platelet count, and neutrophil-to- 
lymphocyte ratio (NLR) (Figure 1). The characteristics of patients in this study cohort are listed in Table 1. The low-SII and high-SII groups consisted of 73 and 106 cases. The rate of the cases with anemia, neutrophilia, thrombocytosis, hypercalcemia, time from diagnosis to treatment $<1$ year, and with poor risk of the IMDC model were higher in the high SII group than those in the low SII group (Table 1).

The median of the observation period was 24 months, and the $50 \%$ OS in the entire cohort was 43.3 months (Figure 2a). Maximum effects of the first-line agents determined based on the RECIST criteria in each group are shown in Table 2. In the high SII group, the rate of cases with complete response (CR) and partial response (PR) was significantly lower ( $\mathrm{p}=0.0394$ ), and that with progressive disease (PD) was significantly higher $(\mathrm{p}=0.0038)$ in the high SII group than those in the low SII group.

The 50\% OS in the high-SII group was 21.4 months, which was significantly worse than that in the low-SII group (49.7 months, $\mathrm{p}<0.0001$, Figure 2b). Multivariate analysis showed that a high SII as well as non-clear histology, hypercalcemia, and time from diagnosis to treatment $<1$ year was an independent predictive factor for a worse OS (Table 3). Next, we constructed a modified IMDC risk model that included the SII instead of the neutrophil count and platelet count. Using this modified IMDC model, all cases were re-classified into four groups of 33, 52, 81, and 13 cases with $50 \%$ OS of $88.8,45.9,29.4$, and 4.8 months, respectively ( $<<0.0001)$, while 102 cases, $57 \%$ of all of them, were classified into the intermediate risk group based on the conventional IMDC model (Figure 3).

\section{Discussion}

In this study, we assessed the impact of the SII for predicting the prognosis of cases with mRCC treated with TKI, and we showed the usefulness of the SII to modify the IMDC risk model. To the best of our knowledge, this study is the first report on the impact of SII for establishing a modified prognostic model.

Several prognostic classifications are available for mRCC [11]. Of these, the Memorial Sloan Kettering Cancer Center (MSKCC) risk model and the IMDC risk model are two of the most widely-used ones. The former was based on data from patients who were enrolled in clinical trials of cytokine therapy, and the latter was derived from patients treated with targeted agents. In both models, patients were 
stratified into 3 categories: favorable, intermediate, and poor risk groups. Also, more than half of the patients were categorized as being in the intermediate risk group.

Because many patients with different prognoses are thought to be included in the same "intermediate risk" group, improvement in risk stratification, especially in the intermediate risk group, is required for both models. Some investigators focused on the relationship between the number of positive risk factors. Tamada et al. [12] and Sella et al. [13] reported that $\mathrm{mRCC}$ patients treated with targeted agents in the intermediate risk of the MSKCC model could be divided into two groups with different prognoses. Others were re-stratified into intermediate risk group patients based on the CRP level [14][15], which has been demonstrated as a predictive factor for prognosis and therapeutic efficacy in many reports [16][17]. In addition to CRP, various reports have demonstrated the association of enhanced inflammatory response with the progression of RCC including peripheral blood markers and indexes constructed from these components. Neutrophils can secrete various growth factors and cytokines, and they are associated with the stimulation of the tumor microenvironment [18]. Lymphocytes can show an antitumoral role through the induction of cytotoxic cell death [19][20], and perioperative lymphopenia was reported to be associated with inferior prognosis in patients with mRCC [21]. NLR is one of the most representative indexes that has been reported with its prognostic impact on mRCC [18][22][23]. According to these reports, Tanaka et al. [24] established an IMDC model modified using NLR. Platelets have been reported to be capable of inducing epithelial-to mesenchymal transition, promoting migration and metastasis, and protecting the autoimmune system from cancer cells [25]. The SII can represent these three peripheral blood parameters involving different molecular mechanisms of cancer cells. Also, previous studies have reported the prognostic impact of the SII for mRCC treated with targeted agents and immune checkpoint inhibitors [26]. Therefore, we first investigated the impact of SII on the prediction of the OS of patients with mRCC treated with targeted therapy. As shown in Table 1, the rates of cases with parameters for poor prognosis were significantly higher than those in the high SII group. Moreover, according to multivariate analysis, the high SII was an independent predictive factor for OS (Table 3). These were consistent with the data from previous studies that have reported the association of elevated SII with 
poor prognosis of patients with malignant diseases, including mRCC [9][10].

Simplicity of risk model is important to use in the real world of clinical practice. Because SII is calculated using neutrophils and platelets which are parameter in IMDC model, and lymphocytes which is measured alongside neutrophils, the modified risk model including SII can be used without any additional examination.

Recently, effectiveness of combination regimens which consists of one TKI and one ICI besides one which includes two ICIs [4] were demonstrated by RCTs [27][28]. While options for first-line therapy for mRCC have increased, it is still unclear how to decide the best one. IMDC model was established based on the data of patients who underwent target therapy, therefore, patients with higher risk in IMDC model are indicated to be resistant to targeted agents. While options for first-line therapy for $\mathrm{mRCC}$ have increased, to clarify how to decide the best one is very important theme. Since risk level of IMDC model is thought to represent the resistance for targeted agents, restratification to more subgroups of this model can help the decision of the therapeutic option.

Previous studies demonstrated the possibilities of candidates for biomarkers derived from blood samples. Investigators have previously focused on many molecules as candidates for serum biomarkers including growth factors [29][30], microRNAs [31], and cell free DNAs [32]. However, clinically applying these molecules as biomarkers for mRCC is difficult currently. However, components of SII are routinely measured in real clinical practice during the management of patients with $\mathrm{mRCC}$ undergoing systemic therapy. Our data indicate the possibility of the SII as a biomarker to enable us to improve the prognostic model for $\mathrm{mRCC}$ in the near future.

The limitation of the present report is that it is just a small, retrospective study. Because the data were derived from real-world clinical practice, some selection bias of patients or therapeutic options is inevitable. In addition, inflammatory factors represent not the sensitivity of targeted agents but the status of progressiveness of $\mathrm{mRCC}$; therefore, they can be affected by other therapeutic options [26][33][34]. Further prospective study with higher volume is required to confirm the impact of the SII for predicting the prognosis of patients with mRCC. 


\section{Conclusions}

We demonstrated the impact of SII for the prediction of the prognosis and modification of risk model in patients with mRCC treated with first-line TKI. The SII is promising as a prognostic factor for $\mathrm{mRCC}$ patients, and this finding might lead to the establishment of some novel therapeutic strategies for $\mathrm{mRCC}$, leading to an era of multiple options including targeted agents or immune checkpoint inhibitors. 


\section{References}

1. Vera-Badillo FE, Templeton AJ, Duran I, et al. Systemic therapy for non-clear cell renal cell carcinomas: a systematic review and meta-analysis. Eur Urol 2015; 67: 740-9.

2. Pal SK, Nelson RA, Vogelzang N. Disease-specific survival in de novo metastatic renal cell carcinoma in the cytokine and targeted therapy era. PLoS One 2013; 8: e63341.

3. Motzer RJ, Escudier B, McDermott DF, et al. Nivolumab versus everolimus in advanced renal-cell carcinoma. N Engl J Med 2015; 373: 1803-13.

4. Motzer RJ, Tannir NM, McDermott DF, et al. Nivolumab plus ipilimumab versus sunitinib in advanced renal-cell carcinoma. N Engl J Med 2018; 378: 1277-90.

5. https://www.nccn.org/professionals/physician_gls/PDF/kidney.pdf

6. Ljungberg B, Bensalah K, Canfield S, et al. EAU guidelines on renal cell carcinoma: 2014 update. Eur Urol 2015; 67: 913-24.

7. Heng DY, Xie W, Regan MM, et al. Prognostic factors for overall survival in patients with metastatic renal cell carcinoma treated with vascular endothelial growth factor-targeted agents: results from a large, multicenter study. J Clin Oncol 2009; 27: 5794-9.

8. Teishima J, Inoue S, Hayashi T, et al. Current status of prognostic factors in patients with metastatic renal cell carcinoma. Int J Urol 2019; 26: 608-17.

9. Zhong JH, Huang DH, Chen ZY. Prognostic role of systemic immuneinflammation index in solid tumors: a systematic review and meta-analysis. Oncotarget 2017; 8: 75381-8.

10. Lolli C, Basso U, Derosa L, et al. Systemic immune-inflammation index predicts the clinical outcome in patients with metastatic renal cell cancer treated with sunitinib. Oncotarget 2016; 7: 54564-71.

11. Motzer RJ, Bacik J, Murphy BA, et al. Interferon-alfa as a comparative treatment for clinical trials of new therapies against advanced renal cell carcinoma. J Clin Oncol 2002; 20: 289-96.

12. Tamada S, Iguchi T, Yasuda S, et al. The difference in the survival rate of patients with metastatic renal cell carcinoma in the intermediate-risk group of the Memorial Sloan Kettering Cancer Center criteria. Oncotarget 2018; 9: 27752-9. 
13. Sella A, Michaelson MD, Matczak E, et al. Heterogeneity of patients with intermediate-prognosis metastatic renal cell carcinoma treated with sunitinib. Clin Genitourin Cancer 2017; 15: 291-9.e1.

14. Teishima J, Kobatake K, Hayashi T, et al. Prognostic significance of C-reactive protein in patients with intermediate-risk metastatic renal cell carcinoma treated with molecular targeted therapy. Oncol lett 2014; 8: 881-5.

15. Takamatsu K, Mizuno R, Omura M, et al. Prognostic value of baseline serum Creactive protein level in intermediate-risk group patients with metastatic renalcell carcinoma treated by first-line vascular endothelial growth factor-targeted therapy. Clin Genitourin Cancer 2018; 16: e927-e33.

16. Teishima J, Kobatake K, Kitano H, et al. The impact of change in serum Creactive protein level on the prediction of effects of molecular targeted therapy in patients with metastatic renal cell carcinoma. BJU Int 2016; 117: E67-74.

17. Teishima J, Kobatake K, Shinmei S, et al. The effect of kinetics of C-reactive protein in the prediction of overall survival in patients with metastatic renal cell carcinoma treated with tyrosine kinase inhibitor. Urol Oncol 2017; 35: 662.e1e7.

18. Templeton AJ, McNamara MG, Šeruga B, et al. Prognostic role of neutrophil-tolymphocyte ratio in solid tumors: a systematic review and meta-analysis. $J$ Natl Cancer Inst 2014; 106: dju124.

19. De Giorgi U, Mego M, Scarpi E,et al. Relationship between lymphocytopenia and circulating tumor cells as prognostic factors for overall survival in metastatic breast cancer. Clin Breast Cancer 2012; 12: 264-9.

20. Minami T, Minami T, Shimizu N, et al. Identification of programmed death ligand 1-derived peptides capable of inducing cancerreactive cytotoxic $\mathrm{T}$ lymphocytes from HLA-A24+ patients with renal cell carcinoma. J Immunother 2015; 38: 285-91.

21. Saroha S, Uzzo RG, Plimack ER, et al. Lymphopenia is an independent predictor of inferior outcome in clear cell renal carcinoma. J Urol 2013; 189 : 454-61.

22. Templeton AJ, Knox JJ, Lin X, et al. Change in neutrophil-to-lymphocyte ratio in response to targeted therapy for metastatic renal cell carcinoma as a prognosticator and biomarker of efficacy. Eur Urol 2016; 70: 358-64.

23. Teishima J, Ohara S, Sadahide K, et al. Impact of neutrophil-to-lymphocyte ratio on effects of targeted therapy for metastatic renal cell carcinoma patients with extrapulmonary metastasis. Can Urol Assoc J 2017; 11: E207-14. 
24. Tanaka N, Mizuno R, Yasumizu Y, et al. Prognostic value of neutrophil-tolymphocyte ratio in patients with metastatic renal cell carcinoma treated with first-line and subsequent second-line targeted therapy: a proposal of the modified-IMDC risk model. Urol Oncol 2017; 35: 39.e19-e28.

25. Schumacher D, Strilic B, Sivaraj KK, et al. Platelet-derived nucleotides promote tumor-cell transendothelial migration and metastasis via P2Y2 receptor. Cancer Cell 2013; 24: 130-7.

26. De Giorgi U, Procopio G, Giannarelli D, et al. Association of systemic inflammation index and body mass index with survival in patients with renal cell cancer treated with nivolumab. Clin Cancer Res 2019; 25: 3839-46.

27. Motzer RJ, Penkov K, Haanen J, et al. Avelumab plus axitinib versus sunitinib for advanced renal-cell carcinoma. N Engl J Med. 2019; 380: 1103-15.

28. Rini BI, Plimack ER, Stus V, et al. Pembrolizumab plus axitinib versus sunitinib for advanced renal-cell carcinoma. N Engl J Med. 2019; 380: 1116-27.

29. Hegde PS, Jubb AM, Chen D, et al. Predictive impact of circulating vascular endothelial growth factor in four phase III trials evaluating bevacizumab. Clin Cancer Res 2013; 19: 929-37.

30. Peña C, Lathia C, Shan M, et al. Biomarkers predicting outcome in patients with advanced renal cell carcinoma: Results from sorafenib phase III Treatment Approaches in Renal Cancer Global Evaluation Trial. Clin Cancer Res 2010; 16 : 4853-63.

31. Gu L, Li H, Cken K, et al. Micro RNAs as prognostic molecular signatures in renal cell carcinoma: a systematic review and meta-analysis. Oncotarget 2015; 6: 32545-60.

32. de Martino M, Klatte T, Haitel A, et al. Serum cell-free DNA in renal cell carcinoma: a diagnostic and prognostic marker. Cancer 2012; 118: 82-90.

33. Teishima J, Ohara S, Shinmei S, et al. Normalization of C-reactive protein levels following cytoreductive nephrectomy in patients with metastatic renal cell carcinoma treated with tyrosine kinase inhibitors is associated with improved overall survival. Urol Oncol 2018; 36: 339.e9-e15.

34. Teishima J, Murata D, Hasegawa Y, et al. C-reactive protein can be used to predict the therapeutic effects of nivolumab in patients with metastatic renal cell carcinoma. Int J Urol 2019; 26: 1076-7. 


\section{Figures and Tables}

Fig. 1. Correlation between systemic immune inflammatory index (SII) and other inflammatory parameters including (a) C-reactive protein (CRP); (b) platelet count; (c) neutrophil count; and (d) neutrophil-to-lymphocyte ratio (NLR).
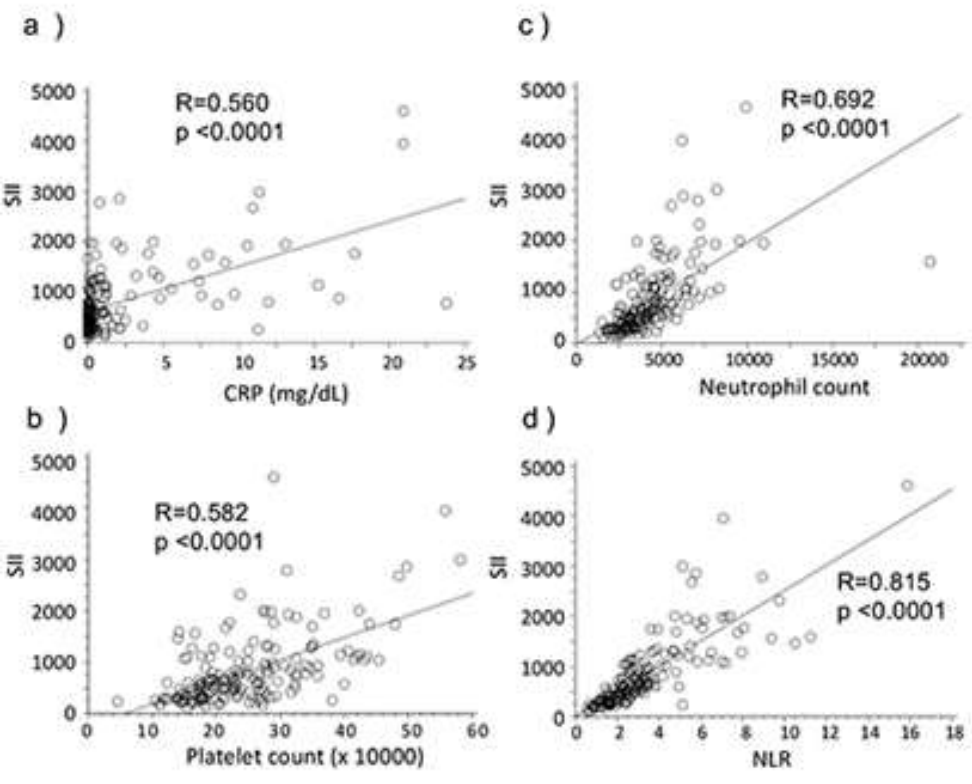

Fig. 2. Overall survival (OS) (a) for all cases and (b) OS stratified based on pretreated systemic immune inflammatory index (SII).

a)

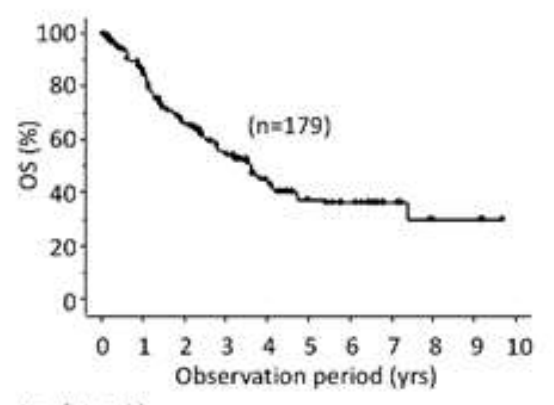

No. of pt. st risk

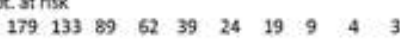

b)

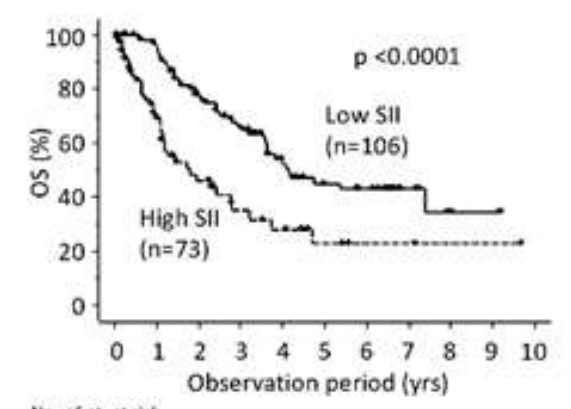

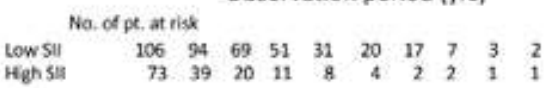


Fig. 3. Overall survival (OS) stratified based on (a) international metastatic renal cell carcinoma database consortium (IMDC) risk model and $(\boldsymbol{b})$ modified version of IMDC risk model consisting of 5 factors including systemic immune inflammatory index (SII).

a)

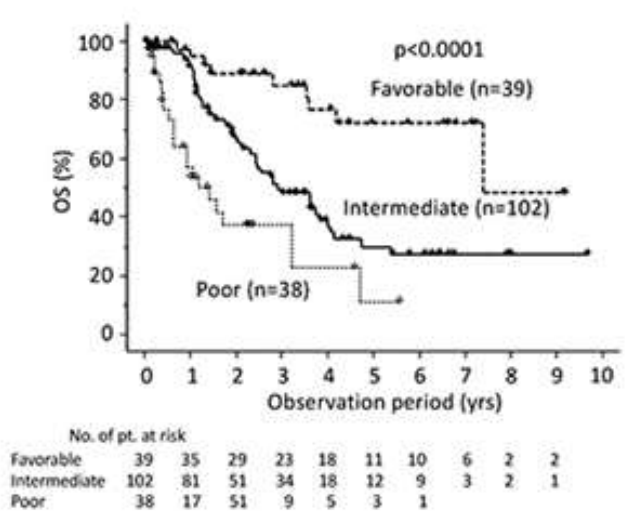

b)

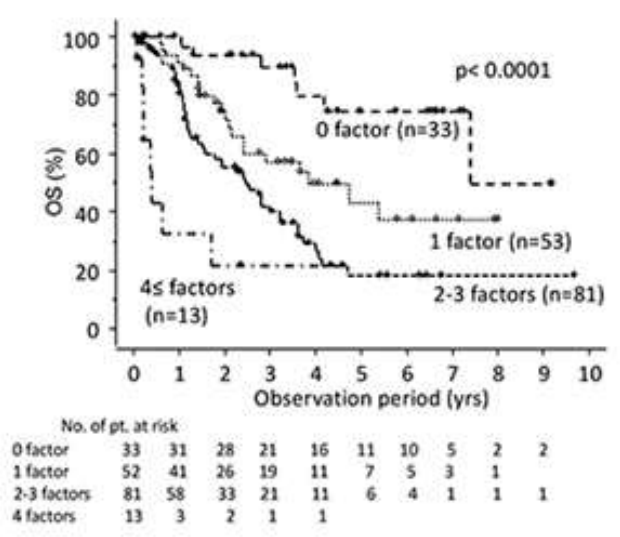

\begin{tabular}{|c|c|c|c|c|}
\hline & $\begin{array}{c}\text { High SII } \\
(\geq 730)\end{array}$ & $\begin{array}{c}\text { Low SII } \\
(<730)\end{array}$ & $\mathbf{p}$ & \\
\hline No. of patients & $(n=73)$ & $(n=106)$ & & $(n=179)$ \\
\hline Age (median) & $64(40-85)$ & $67(40-85)$ & 0.1624 & $65(40-85)$ \\
\hline \multicolumn{5}{|l|}{ Sex $(\%)$} \\
\hline Male & $61(83.6)$ & $84(79.2)$ & 0.4694 & $145(81.0)$ \\
\hline Female & $12(16.4)$ & $22(20.8)$ & & $34(19.0)$ \\
\hline \multicolumn{5}{|c|}{ Histological type (\%) } \\
\hline Clear & $61(83.6)$ & $96(90.6)$ & 0.1607 & $157(87.7)$ \\
\hline Non-clear & $12(16.4)$ & $10(9.4)$ & & $22(12.3)$ \\
\hline \multicolumn{5}{|l|}{ KPS (\%) } \\
\hline$\geq 80 \%$ & $65(89.0)$ & $101(95.3)$ & 0.1138 & $166(92.7)$ \\
\hline$<80 \%$ & $8(11.0)$ & $5(4.7)$ & & $13(7.3)$ \\
\hline \multicolumn{5}{|l|}{ Anemia $(\%)$} \\
\hline$(-)$ & $45(61.6)$ & $36(34.0)$ & 0.0003 & $81(45.3)$ \\
\hline
\end{tabular}



Systemic immune-inflammation index in mRCC

\begin{tabular}{|c|c|c|c|c|}
\hline$(+)$ & $28(38.4)$ & $70(66.0)$ & & $98(54.7)$ \\
\hline \multicolumn{5}{|l|}{ Neutrophilia (\%) } \\
\hline$(-)$ & $50(68.5)$ & $100(94.3)$ & $<0.0001$ & $150(83.8)$ \\
\hline$(+)$ & $23(31.5)$ & $6(5.7)$ & & $29(16.2)$ \\
\hline \multicolumn{5}{|l|}{ Thrombocytosis (\%) } \\
\hline$(-)$ & $51(69.9)$ & $104(98.1)$ & $<0.0001$ & $155(86.6)$ \\
\hline$(+)$ & $22(30.1)$ & $2(1.9)$ & & $24(13.4)$ \\
\hline \multicolumn{5}{|l|}{ Hypercalcemia (\%) } \\
\hline$(-)$ & $66(90.4)$ & $106(100)$ & 0.0011 & $172(96.1)$ \\
\hline$(+)$ & $7(9.6)$ & $0(0)$ & & $7(3.9)$ \\
\hline \multicolumn{5}{|l|}{ Metastatic organs $(\%)$} \\
\hline 1 & $6(42.9)$ & $8(30.8)$ & 0.4446 & $14(35.0)$ \\
\hline$\geq 2$ & $8(57.1)$ & $18(69.2)$ & & $26(65.0)$ \\
\hline \multicolumn{5}{|l|}{$\begin{array}{l}\text { Time from diagnosis to } \\
\text { treatment }(\%)\end{array}$} \\
\hline$\geq 1$ year & $20(27.4)$ & $45(42.5)$ & 0.0396 & $65(36.3)$ \\
\hline$<1$ year & $53(72.6)$ & $61(57.5)$ & & $114(63.7)$ \\
\hline \multicolumn{5}{|l|}{ IMDC risk } \\
\hline Favorable & $8(11.0)$ & $31(29.3)$ & 0.0036 & $39(21.8)$ \\
\hline Intermediate & $31(42.5)$ & $71(67.0)$ & & $102(57.0)$ \\
\hline Poor & $34(46.6)$ & $4(3.8)$ & $<0.0001$ & $38(21.2)$ \\
\hline \multicolumn{5}{|l|}{ Metastatic site } \\
\hline Lung & $48(65.8)$ & $80(75.4)$ & & $128(71.5)$ \\
\hline Lymphnode & $25(34.3)$ & $30(28.3)$ & & $55(30.7)$ \\
\hline Liver & $9(12.3)$ & $13(12.3)$ & & $22(12.3)$ \\
\hline Bone & $20(27.4)$ & $26(24,5)$ & & $46(25.7)$ \\
\hline Pancreas & $2(2.7)$ & $7(6.6)$ & & $9(5.0)$ \\
\hline Adrenal gland & $7(9.6)$ & $7(6.6)$ & & $14(7.8)$ \\
\hline Contralateral kidney & $5(6.8)$ & $10(9.4)$ & & $15(8.4)$ \\
\hline Brain & $3(4.1)$ & $4(3.7)$ & & $7(3.9)$ \\
\hline Soft tissue & $4(5.5)$ & $4(3.7)$ & & $8(4.5)$ \\
\hline \multicolumn{5}{|l|}{ Prior nephrectomy, n (\%) } \\
\hline Radical & $28(38.4)$ & $65(61.3)$ & 0.0025 & $93(52.0)$ \\
\hline Cytoreductive & $33(45.2)$ & $34(32.1)$ & & $67(37.4)$ \\
\hline None & $12(16.4)$ & $7(6.6)$ & 0.0358 & $19(10.6)$ \\
\hline
\end{tabular}




\begin{tabular}{|c|c|c|l|c|}
\hline $1^{\text {st }}$ line agents, $\mathrm{n}(\%)$ & & & & \\
\hline Sunitinib & $55(75.3)$ & $59(55.7)$ & & $114(63.7)$ \\
\hline Pazopanib & $5(6.8)$ & $7(6.6)$ & & $12(6.7)$ \\
\hline Ssorafenib & $13(17.8)$ & $38(35.9)$ & & $51(28.5)$ \\
\hline Axitinib & $0(0)$ & $2(1.9)$ & & $2(1.1)$ \\
\hline
\end{tabular}

KPS: Karnofsky performance status, IMDC: International Metastatic Renal Cell Carcinoma Database Consortium.

\begin{tabular}{|l|c|c|c|}
\hline \multicolumn{4}{|c|}{ Table 2. Best objective response of first-line agents in each group } \\
\hline & High SII & Low SII & Total \\
\hline No. of patients & $(\mathrm{n}=64)$ & $(\mathrm{n}=88)$ & $(\mathrm{n}=152)$ \\
\hline CR/PR & $8(12.5)$ & $23(26.1)$ & $31(20.4)$ \\
\hline SD & $40(62.5)$ & $58(65.9)$ & $98(64.5)$ \\
\hline PD & $16(25.0)$ & $7(8.0)$ & $23(15.1)$ \\
\hline
\end{tabular}

CR: complete response; PD: progressive disease; PR: partial response; SD: stable disease; SII: systemic immune inflammatory index. 


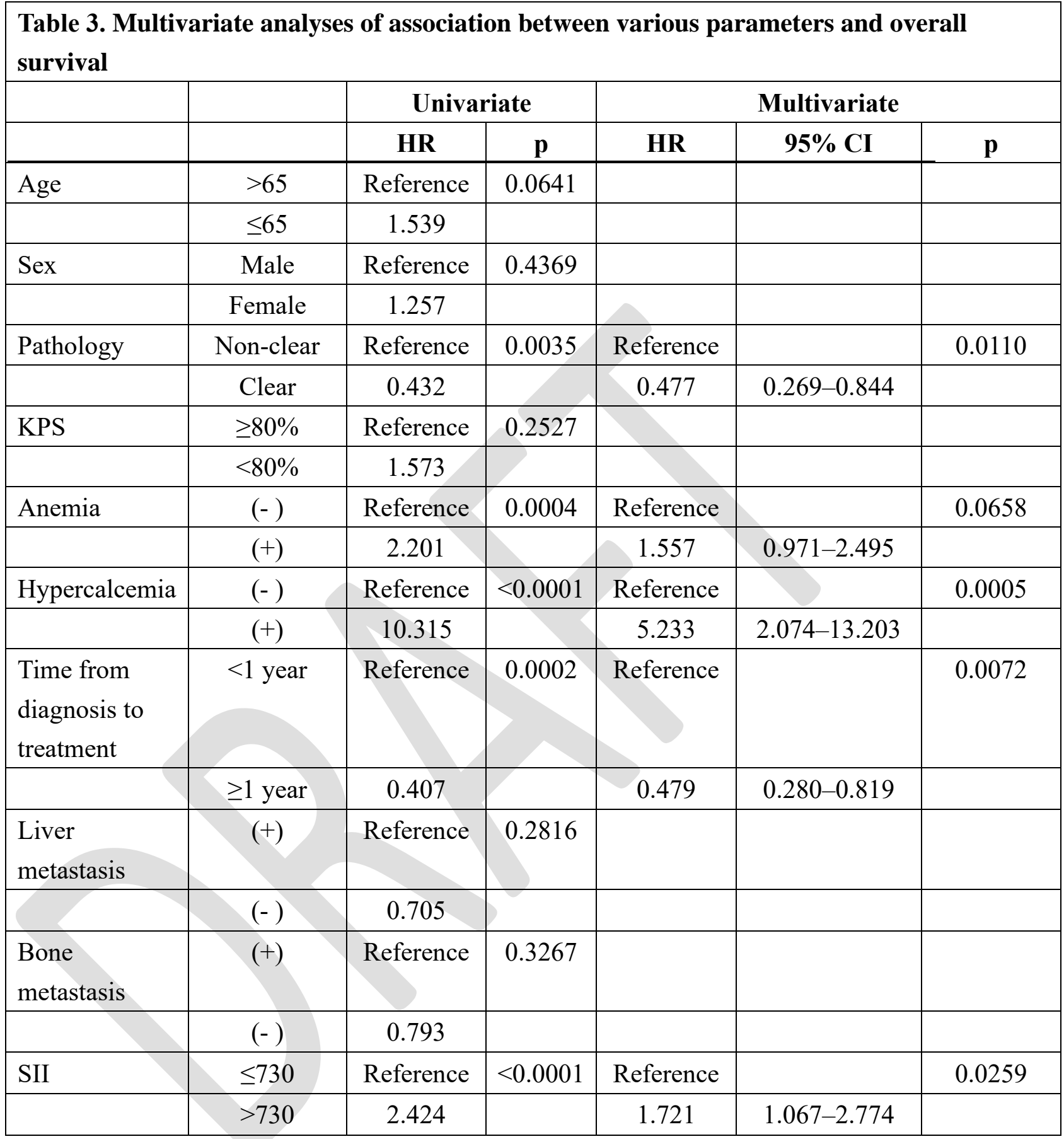

CI: confidence interval; HR: hazard ratio; KPS: Karnofsky Performance Status; SII: systemic immune inflammatory index. 